\title{
Evaluating the Diffusion Approximation Capability on the Integral Pressurized Water Reactor (IPWR) Core Calculation
}

\author{
H. Ardiansyah ${ }^{1 *}$, M. R. Oktavian ${ }^{2,3}$ \\ ${ }^{I}$ Department of Nuclear Engineering and Radiological Sciences, University of Michigan, 2355 Bonisteel Blvd, \\ Ann Arbor, MI 48109, United States of America \\ ${ }^{2}$ School of Nuclear Engineering, Purdue University, 516 Northwestern Ave., West Lafayette, IN 47906, United States of America \\ ${ }^{3}$ Department of Nuclear Engineering and Engineering Physics, Faculty of Engineering, Universitas Gadjah Mada, \\ Jl. Grafika No. 2, Yogyakarta 55281, Indonesia
}

\section{ARTICLE INFO}

Article history:

Received 13 December 2019

Received in revised form 18 March 2021

Accepted 19 March 2021

\section{Keywords:}

Diffusion

Two-step method

IPWR

PARCS

Serpent

\begin{abstract}
A B S T R A C T
Diffusion approximation is an important approximation used to model a nuclear reactor core with a quite good accuracy and less computational cost. This approximation has been used widely around the globe for various kinds of nuclear reactors. This diffusion approximation is applied in a two-step method, a method combining a high fidelity transport code and low fidelity diffusion code. Meanwhile, innovations in the nuclear core model continue to make the nuclear reactor core safer, more robust, and smaller. The trend of creating smaller and more modular reactor core is emerging in the last ten years. These innovations will affect the core modeling system. Consequently, for smaller reactors, it is important to evaluate the capability of diffusion approximation if one wants to use a computationally cheaper method to model the reactor core. In this paper, neutron diffusion calculation for $160 \mathrm{MWth}$ integral pressurized water reactor (IPWR) core was conducted using the PARCS nodal diffusion code employing the few-group spatially homogenized cross-sections generated by the Serpent Monte Carlo code. Due to its capability to model any reactor geometry in the high-resolution calculation, the results from Serpent were also used as a reference. Two important parameters are compared between PARCS and Serpent: effective neutron multiplication factor and core power distribution. For the full IPWR core model, a discrepancy of $564 \mathrm{pcm}$ between PARCS and Serpent $k_{\text {eff }}$ was observed, while the radial power distribution had a maximum error of $4.71 \%$. It can be said, to some extent, that the diffusion approximation can be applied to IPWR core analysis. However, further improvement is indeed required if one wants more accurate results with low computational costs.
\end{abstract}

(C) 2021 Atom Indonesia. All rights reserved

\section{INTRODUCTION}

In nuclear reactor physics, the behavior of the whole reactor is closely related to the neutron movement inside the reactor core that drives the fission reaction in the fuel. Since neutrons are neutral particles, they will travel in straight lines without any deviation, except when they collide and interact with certain matters. Having this characteristic, the neutronic phenomenon that occurs in the nuclear reactor can be exactly explained with

\footnotetext{
*Corresponding author.

E-mail address: harun@umich.edu

DOI: https://doi.org/10.17146/aij.2021.1013
}

neutron transport theory that governs how neutrons are transported through various media.

Two different types of methods are usually followed to solve for neutron flux in the transport equation: deterministic and stochastic. The deterministic method uses an approximation to discretize all independent variables and numerically solve the Boltzmann transport equation. On the other hand, the stochastic solutions, usually called Monte Carlo methods, use brute force to statistically model neutron behaviors in the reactor instead of solving the transport equation [1].

Both methods, especially the Monte Carlo method, require an extensive amount of computational resources to simulate neutron 
behaviors in typical nuclear reactors. As a result, several researchers use further approaches using diffusion approximations to simplify the problems, especially ones that involve time-dependent transient calculation. Approaches to combine both deterministic and stochastic methods are developed to solve the computational challenges of criticality calculation. The resulting method is called the two-step method. As the name suggests, these methods combine the stochastic Monte Carlo codes, which are used for generating homogenized crosssections and neutron flux distribution, and the deterministic codes, which are then used for criticality safety calculation for steady-state or even transient conditions using data generated by the Monte Carlo codes.

The recent development of nuclear technology, especially in the last decades, shows several trends toward smaller-size reactors called small modular reactors (SMRs) [2,3]. The smaller economic investment and faster construction time of these reactors make them interesting options soon.

This work has a purpose to test the performance of the diffusion method to use in the neutronic analysis of the 160 MWth IPWR (integral pressurized water reactor). This reactor is used as a reference case because of its applicability and the capability to use natural uranium in the MOX fuelmaking the IPWR an interesting choice for future advanced small-sized nuclear power plant [4]. Hence, finding an efficient method to analyze this reactor is important before one decides to build it in the future.

\section{THEORY}

In nuclear reactor physics, the behavior of the whole reactor is closely related to the neutron movement inside the reactor core that drives the fission reaction in the fuel. Since neutrons are neutral particles, they will travel in straight lines without any deviation, except when they collide and interact with certain matters. Having this characteristic, the neutronic phenomenon that occurs in the nuclear reactor can be exactly explained with neutron transport theory that governs how neutrons are transported through various media.

The neutron transport theory is represented in an equation called the Boltzmann transport equation. The equation describes the neutron balance in a system: the rate of neutron change in time is equal to the rate of neutron gain subtracted by the rate of neutron loss. The neutron gain in the reactor can be from the fission production (prompt or delayed), scattered neutrons that enter the system, and external sources. The neutron loss in the system can be caused by the collision due to an interaction with matters and neutron leakage out of the system.

Even though the transport equation seems to be quite straightforward, the process to solve it is unfortunately not easy at all. Especially for a large, time-dependent model, the complexity makes it almost impossible to model in the high-resolution neutron transports.

To further explain this, suppose we have a time-dependent neutron transport equation written in Eq. (1).

$$
\begin{aligned}
\frac{1}{v} \frac{\partial \psi(\boldsymbol{x}, \widehat{\mathbf{\Omega}}, E, t)}{\partial t} & +\widehat{\mathbf{\Omega}} \cdot \nabla \psi(\boldsymbol{x}, \widehat{\mathbf{\Omega}}, E, t) \\
& +\Sigma_{t}(\boldsymbol{x}, E) \psi(\boldsymbol{x}, \widehat{\mathbf{\Omega}}, E, t) \\
& =\int_{0}^{\infty} \int_{4 \pi} \Sigma_{s}\left(\boldsymbol{x}, \widehat{\mathbf{\Omega}^{\prime}} \cdot \widehat{\mathbf{\Omega}}, E^{\prime}\right. \\
& \rightarrow E) \psi\left(\boldsymbol{x}, \widehat{\boldsymbol{\Omega}}^{\prime}, E^{\prime}, t\right) d \Omega^{\prime} d E^{\prime} \\
& +\frac{\chi(E)}{4 \pi} \int_{0}^{\infty} \int_{4 \pi} v \Sigma_{f}\left(\boldsymbol{x}, E^{\prime}\right. \\
& \rightarrow E) \psi\left(\boldsymbol{x}, \widehat{\mathbf{\Omega}}^{\prime}, E^{\prime}, t\right) d \Omega^{\prime} d E^{\prime}
\end{aligned}
$$

Where:

$$
\begin{aligned}
\boldsymbol{x} & =\text { space vector }(x, y, z) \\
\widehat{\mathbf{\Omega}} & =\text { directional vector space vector }(\mu, \gamma) \\
E & =\text { energy } \\
t & =\text { time } \\
\psi & =\text { neutron angular flux } \\
\Sigma_{x} & =\text { macroscopic cross-sections of reaction } x \\
& \text { (total, scattering, or fission) } \\
v & =\text { neutron speed } \\
\chi & =\text { fission spectrum } \\
v & =\text { average neutron produced from fission }
\end{aligned}
$$

Equation (1) can then be deterministically solved by applying several approximations. The most common approximation is the P1 approximation which then leads to diffusion approximation. The final equation that is solved in the diffusion equation is laid out in Eq. (2).

$$
-\nabla \cdot D \nabla \phi+\Sigma_{a} \phi=\frac{1}{k} v \Sigma_{f} \phi
$$

Equation (2) is the $k$-eigenvalue diffusion equation for the steady-state condition. The fundamental assumption used in the diffusion equation that the angular flux is a linear function of $\widehat{\mathbf{\Omega}}$ will only be valid if the spatial derivatives of the scalar flux are negligible. This is true for an optically thick system where scattering reaction is dominant. For this reason, the diffusion equation is pretty accurate to model a typical large commercial nuclear reactor core where the spatial gradients are not prominent due to its large size and the low number of absorbing media. 


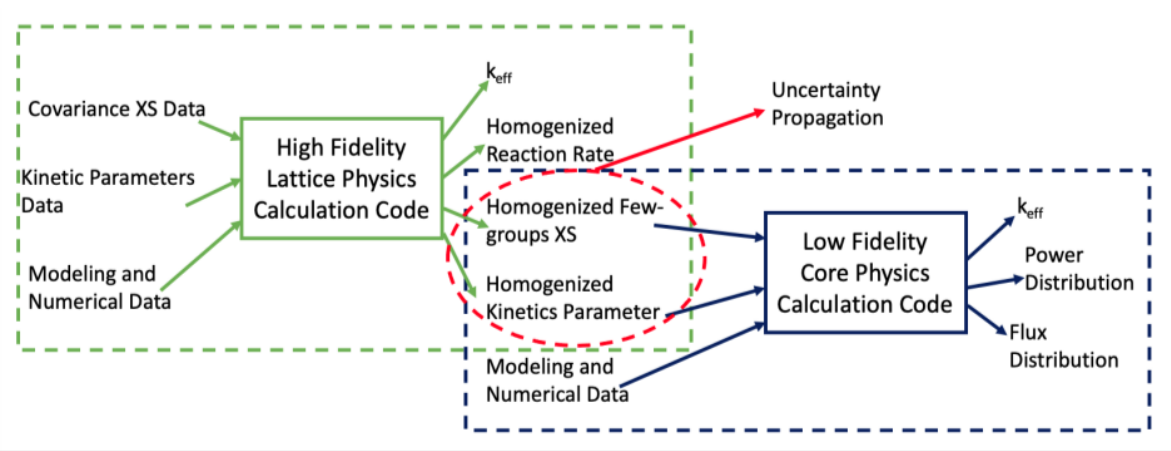

Fig. 1. Two-step method.

In the common practice of nuclear reactor core simulation, a nodal approach is used, i.e., modeling the reactor in assembly-wise or pin-wise node, then coupling all the nodes together to obtain full core neutronic behavior of the reactor [4]. Thus, the diffusion equation used in this approach is given in Eq. (3).

$$
\begin{aligned}
-D_{i, g} \nabla^{2} \phi_{i, g}+ & \Sigma_{r, i, g} \phi_{i, g} \\
& =\frac{\chi_{i, g}}{k_{e f f}} \sum_{g^{\prime}} v \Sigma_{f, i, g^{\prime}} \phi_{i, g^{\prime}} \\
& +\sum_{g^{\prime}} \Sigma_{s, i, g^{\prime} \rightarrow g} \phi_{i, g^{\prime}}
\end{aligned}
$$

In (3), $i$ is the node index where the diffusion equation is solved. Since every individual node is modeled homogenously, the nodal few-group cross-sections are obtained beforehand from the lattice physics calculation, either deterministic or Monte Carlo.

The spatial homogenization and energy group condensation to obtain the nodal cross-sections are usually done by preserving the related reaction rates from the lattice physics calculation used. The equation for this is written as in Eq. (4).

$$
\begin{aligned}
& \Sigma_{g}^{\text {hom }}=\left\langle\Sigma_{g}\right\rangle_{\text {node }} \\
& =\frac{\int_{R} \int_{E_{g}}^{E_{g}-1} \Sigma(x, E) \phi(\boldsymbol{x}, E) d E d V}{\int_{R} \int_{E_{g}}^{E_{g-1}} \phi(\boldsymbol{x}, E) d E d V}
\end{aligned}
$$

If the homogenous group constants are generated using Monte Carlo methods, the reaction rates and fluxes can be tallied along with the calculation. The tally is conducted for every spatial node and energy group bin to obtain the spatially homogenous, group-condensed crosssection data.

Combining the lattice transport calculation to obtain the spatially homogeneous, group-condensed cross-section data and nodal diffusion to obtain the reactor core calculation has been widely performed in light water reactor core analysis. This procedure is called the two-step method. The two-step procedure consists of an assembly transport calculation with a lattice physics code, and a reactor core calculation with a nodal diffusion code. The diagram in Fig. 1 shows the general process of how the two-step method calculation is conducted. The few-group constants, including diffusion coefficients, are generated from the assembly calculation results.

Once the assembly calculation is done, the cross-sections are spatially homogenized, and a critical spectrum calculation is performed in order to take into account the neutron leakages of the lattice. The diffusion coefficient is also generated through the critical spectrum calculation [5]. Using the two-step method, the computational cost of the core calculation can be reduced while maintaining the accuracy of the calculation.

\section{METHODOLOGY}

This research is conducted based on the NuScale integral PWR model. Some simplification was introduced, mainly in the material definition. The material and geometry data of IPWR is presented in Table 1

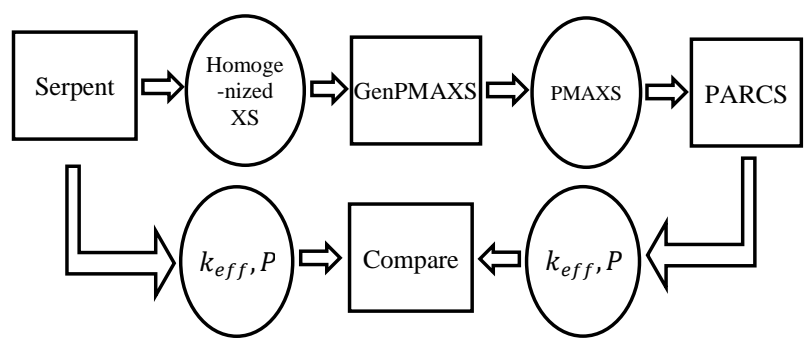

Fig. 2. Procedures employed in this research.

The procedure of this research is illustrated in Fig. 2. The Serpent Monte Carlo code is used to generate nodal cross-sections for PARCS nodal diffusion code and to produce reference results for this work [6,7]. The capability of Serpent to model every detailed part of nuclear reactor design in high resolution and accuracy makes its output suitable as a reference result when experimental data is not available. 
The Serpent Monte Carlo Code, developed at VTT Technical Research Centre of Finland, uses Monte Carlo methods to perform the lattice physics calculation in the reactor and, furthermore, to obtain homogenized cross-sections to use in nodal diffusion calculations [8-10]. Unlike deterministic methods, Monte Carlo methods do not solve the neutron transport equation; rather, they simulate individual particles (neutrons) and record several aspects of their average behavior to obtain results intended by the users. The accuracy of the Monte Carlo calculation depends highly on the number of particle histories included in the calculation, causing this method to be very cost-extensive in terms of computation resources if one wants to obtain an accurate result. Nevertheless, Monte Carlo methods are reliable for solving arbitrary and complicated three-dimensional problems because there are no averaging approximations required in space, energy, and time.

The main job of Serpent is to generate the nodal cross-sections and nodal flux distributions that are then used by PARCS. PARCS uses the fewgroup homogenized nodal cross-sections generated by Serpent and converted by GenPMAXS to perform nodal diffusion calculation on the IPWR model [11]. The nodal calculation is utilized using the nodal expansion method (NEM) for the multigroup diffusion equation. The calculation is further accelerated using the coarse-mesh finite difference (CMFD) method in PARCS [12].

The data of material and geometry used in the Serpent for Monte Carlo calculation is described in Table $1[13,14]$.

The fuel assembly of the IPWR model is described in Fig. 3 and the core geometry axial and radial cross-sections are described in Figs. 4 and 5.

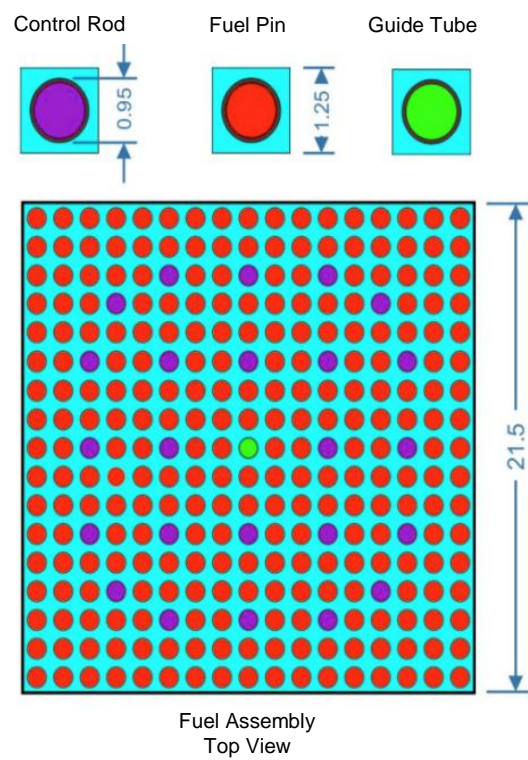

Fig. 3. Top view of Fuel Assembly in $\mathrm{cm}$.
Table 1. Material and geometry data of IPWR.

\begin{tabular}{|c|c|c|}
\hline Properties & Unit & Value \\
\hline \multicolumn{3}{|c|}{ Core } \\
\hline Core radius & $\mathrm{cm}$ & 82.5 \\
\hline Number of assemblies & - & 37 \\
\hline Thermal power & MW & 160 \\
\hline \multicolumn{3}{|c|}{ Fuel Assembly (FA) } \\
\hline Fuel type & - & $\mathrm{PuO}_{2}-\mathrm{UO}_{2}$ \\
\hline${ }^{235} \mathrm{U}$ enrichment & - & $0.72 \%$ \\
\hline $\mathrm{Pu}-\mathrm{U}$ Ratio & - & $12 \%$ \\
\hline Assembly model & - & $\begin{array}{c}\text { Square Array } \\
17 \times 17 \text { pins }\end{array}$ \\
\hline Number of fuel rods & - & 264 \\
\hline Structure material & - & SS 304L \\
\hline FA pitch & $\mathrm{cm}$ & 21.50364 \\
\hline Coolant & - & $\mathrm{H}_{2} \mathrm{O}$ \\
\hline \multicolumn{3}{|c|}{ Fuel Rod } \\
\hline Cladding outer diameter & $\mathrm{cm}$ & 0.94996 \\
\hline Gap thickness & $\mathrm{cm}$ & 0.01651 \\
\hline Fuel diameter & $\mathrm{cm}$ & 0.81153 \\
\hline Gap material & - & Helium \\
\hline Cladding material & - & Zircalloy-4 \\
\hline Fuel rod pitch & $\mathrm{cm}$ & 1.25984 \\
\hline Fuel rod height & $\mathrm{cm}$ & 200 \\
\hline \multicolumn{3}{|c|}{ Reflector } \\
\hline Reflector material & - & SS 304L \\
\hline Axial reflector thickness & $\mathrm{cm}$ & 12.5 \\
\hline Radial reflector thickness & $\mathrm{cm}$ & 14 \\
\hline \multicolumn{3}{|c|}{ Thermal-hydraulic Parameters } \\
\hline Pressure & psia & 1450 \\
\hline Fuel pin average temperature & K & 772.04 \\
\hline Gap average temperature & $\mathrm{K}$ & 739.21 \\
\hline $\begin{array}{l}\text { Moderator average } \\
\text { temperature }\end{array}$ & K & 645.57 \\
\hline Cladding average temperature & K & 557.04 \\
\hline Reflector average temperature & $\mathrm{K}$ & 557.04 \\
\hline
\end{tabular}

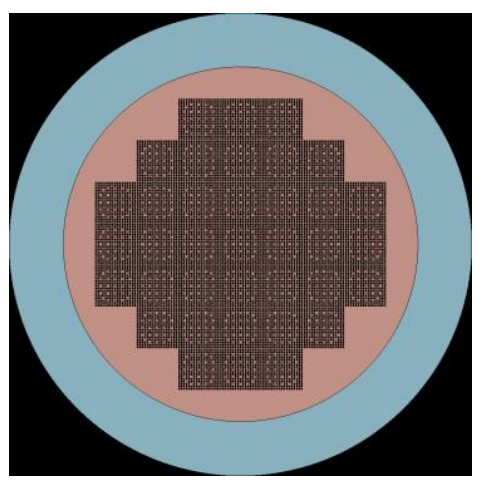

Fig. 4. Radial core geometry cross-section generated by Serpent.

IPWR core modeling is done in multiple stages, starting from 2D basic assemblies. 
Then, the modeling is continued into a $3 \mathrm{D}$ single assembly, 2D color set, and final modeling that includes all the features in the full core model. In this case, Serpent uses eight neutron groups defined using a predefined neutron group (CASMO eight neutron groups) that is included in Serpent. The group structure defined for the group constant generation is tabulated in Table 2. By using neutron populations of $1,000,000$ it is expected that standard deviations are less than $10 \mathrm{pcm}$. For full core calculation, the boundary condition that is applied to the model is a vacuum boundary condition. For other models, the boundary condition used is reflective.

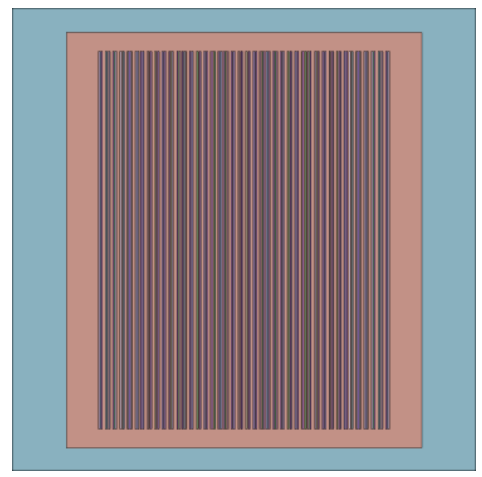

Fig. 5. Axial core geometry cross-section generated by Serpent.

Table 2. Group structure for group constant generation.

\begin{tabular}{cc}
\hline Group & Energy $(\mathrm{MeV})$ \\
\hline 0 & $1.00 \times 10^{1}$ \\
1 & $8.21 \times 10^{-1}$ \\
2 & $5.53 \times 10^{-3}$ \\
3 & $4.00 \times 10^{-6}$ \\
4 & $6.25 \times 10^{-7}$ \\
5 & $2.80 \times 10^{-7}$ \\
6 & $1.40 \times 10^{-7}$ \\
7 & $5.80 \times 10^{-8}$ \\
8 & $1.00 \times 10^{-11}$ \\
\hline
\end{tabular}

Since this research employed steady-state calculation in PARCS, a comparison of $k_{\text {eff }}$ and power distribution in the reactor core with Serpent result is needed $[15,16]$. The capability of diffusion approximation to this problem can be judged by the number of discrepancies in both parameters compared to the reference solution.

\section{RESULTS AND DISCUSSION}

\section{$\boldsymbol{k}_{\text {eff }}$ comparison}

The results of the Monte Carlo calculation conducted in Serpent and nodal diffusion in PARCS for the two-dimensional (2D) single infinite assembly model are shown in Table 3.
Table 3. Single infinite 2D assembly results.

\begin{tabular}{|c|c|c|c|}
\hline \multicolumn{2}{|c|}{ Serpent } & \multirow{2}{*}{$\begin{array}{c}\text { PARCS } \\
k_{\text {eff }} \\
\end{array}$} & \multirow{2}{*}{ Error (pcm) } \\
\hline$k_{e f f}$ & STDEV (pcm) & & \\
\hline 1.22088 & 11 & 1.22088 & 0 \\
\hline
\end{tabular}

From the result, using neutron population of $1,000,000$ and reflective boundary conditions in all directions, it can be observed that the Serpent model and PARCS model showed the same value. It shows that the diffusion method is perfectly accurate for this model. This can be understood since this model employed an infinite repeating assembly model in all directions, thus there is no heterogeneity between one assembly and others, and therefore the nodal diffusion method is exact for this case.

For 3D single assembly, the calculation result is described in Table 4.

Table 4. 3D single assembly results.

\begin{tabular}{|c|c|c|c|}
\hline \multicolumn{2}{|c|}{ Serpent } & \multirow{2}{*}{$\frac{\text { PARCS }}{k_{\text {eff }}}$} & \multirow{2}{*}{ Error (pcm) } \\
\hline$k_{\text {eff }}$ & STDEV (pcm) & & \\
\hline 1.20979 & 1.7 & 1.20935 & 44 \\
\hline
\end{tabular}

Compared to the 2D assembly model, the 3D single assembly model involved finite axial meshes. By having this definition, the axial geometry will cause neutron diffusion approximation to act differently from the 2D assembly model which has an infinite region in all directions due to the reflective boundary. The probability of neutron leakage in the axial direction will be considered in this model. From the result, it is known that there is a difference between the Monte Carlo stochastic calculation and the diffusion approximation. Hence, some small differences, $44 \mathrm{pcm}$, between PARCS and the Serpent results, can be seen.

The result of the full core assembly model is presented in Table 5.

Table 5. $k_{\text {eff }}$ comparison of Serpent and PARCS.

\begin{tabular}{|c|c|c|c|}
\hline \multicolumn{2}{|c|}{ Serpent } & \multirow{2}{*}{$\begin{array}{c}\text { PARCS } \\
k_{\text {eff }}\end{array}$} & \multirow{2}{*}{ Error (pcm) } \\
\hline $\boldsymbol{k}_{\text {eff }}$ & STDEV (pcm) & & \\
\hline 1.07938 & 2.1 & 1.073743 & 563.70 \\
\hline
\end{tabular}

The results show that the difference between Serpent and PARCS in a 3D full core IPWR model is relatively large. However, this result is expected because the 3D full core model is very heterogeneous; therefore, it will have some discontinuity effects, especially in the core regionreflector boundaries. 
Another aspect that needs to be considered is the size of the core itself. This full core model has a diameter of $165 \mathrm{~cm}$ and a height of $200 \mathrm{~cm}$. Compared with any other PWR model in existence, this model is a small and compact model of PWR. This size difference will have an impact on how diffusion approximation is accurate to calculate the criticality of the core. The smaller the core is, the higher the neutron leakage probability becomes. This will result in an inaccuracy in the diffusion approximation. It is different from a typical PWR that has a diameter of 3-6 meters. The neutron leakage probability will be smaller and thus make the diffusion approximation more accurate.

\section{Power comparison}

The power comparison of this IPWR model is described in axial and radial positions. For axial results, power distribution 3D single assembly and full core assemblies power distribution is obtained to be analyzed. For radial results, the power distribution of the full core model is obtained to be analyzed.

The 3D single assembly axial power distribution is described in Table 6.

Table 6. 3D single assembly power comparison.

\begin{tabular}{|c|c|c|c|}
\hline \multirow{2}{*}{$\begin{array}{c}\text { Axial } \\
\text { Midpoint } \\
\text { (cm) }\end{array}$} & \multicolumn{2}{|c|}{ Power (MW) } & \multirow{2}{*}{$\begin{array}{c}\text { Difference } \\
(\%)\end{array}$} \\
\hline & SERPENT & PARCS & \\
\hline 5 & 0.084252 & 0.0897387 & 6.51 \\
\hline 15 & 0.112999 & 0.1096326 & 2.98 \\
\hline 25 & 0.151955 & 0.1498528 & 1.38 \\
\hline 35 & 0.187704 & 0.1859645 & 0.93 \\
\hline 45 & 0.21954 & 0.2184002 & 0.52 \\
\hline 55 & 0.247197 & 0.2465111 & 0.28 \\
\hline 65 & 0.269889 & 0.2696486 & 0.09 \\
\hline 75 & 0.287394 & 0.2873801 & 0.00 \\
\hline 85 & 0.29902 & 0.2994894 & 0.16 \\
\hline 95 & 0.304678 & 0.3055441 & 0.28 \\
\hline 105 & 0.30466 & 0.3055441 & 0.29 \\
\hline 115 & 0.298338 & 0.2994894 & 0.39 \\
\hline 125 & 0.286278 & 0.2873801 & 0.38 \\
\hline 135 & 0.26857 & 0.2696486 & 0.40 \\
\hline 145 & 0.245682 & 0.2465111 & 0.34 \\
\hline 155 & 0.217788 & 0.2184002 & 0.28 \\
\hline 165 & 0.185454 & 0.1859645 & 0.28 \\
\hline 175 & 0.149455 & 0.1498528 & 0.27 \\
\hline 185 & 0.110428 & 0.1096326 & 0.72 \\
\hline 195 & 0.0862054 & 0.0897387 & 4.10 \\
\hline
\end{tabular}

Axially, the obtained power distribution of 3D assembly is similar for Serpent and PARCS. The highest difference is located in the axial boundaries. This can be caused by the discontinuity of flux in the boundaries that become the limitations of diffusion approximation. In the axial midpoint at $75 \mathrm{~cm}$, power distribution calculations by Serpent and PARCS show the same result. Therefore, diffusion approximation is accurate in the reactor mesh, but there is a discontinuity factor that caused the approximation to be inaccurate in the boundaries.

Axial power distribution for the full core model is described in Table 7.

Table 7. Axial power comparison.

\begin{tabular}{ccrc}
\hline $\begin{array}{c}\text { Axial } \\
\text { Midpoint } \\
(\mathbf{c m})\end{array}$ & \multicolumn{2}{c}{ Power (MW) } & Difference \\
\cline { 2 - 3 }$(\boldsymbol{\%})$
\end{tabular}

From the result, it is obtained that the difference between Monte Carlo and nodal diffusion approximation of axial power distribution in the full core model is slightly different from the 3D single assembly. For the full core model, the difference in the boundaries is lower than the 3D single assembly. This is caused by the number of assemblies in the full core model. In the full core model, 37 fuel assemblies are used. This could help to reduce the discontinuity factor in the boundaries. Overall, the difference between meshes in the full core model has a small standard deviation due to the number of fuel assemblies calculated which helps to make the diffusion approximation more accurate. 
The power distribution of radial full core in Serpent and PARCS are described in Tables 8 and 9, while the difference between Serpent and PARCS results is described in Table 10.

Table 8. Full core radial power (MW) Serpent (normalized).

\begin{tabular}{rrrrrrrrr}
\hline $\mathbf{y} \mathbf{x}$ & $\mathbf{1}$ & \multicolumn{1}{c}{$\mathbf{2}$} & \multicolumn{1}{c}{$\mathbf{3}$} & $\mathbf{4}$ & $\mathbf{5}$ & $\mathbf{6}$ & \multicolumn{1}{c}{$\mathbf{7}$} \\
\hline 1 & 0 & 0 & 1.1161 & 3.2670 & 1.1172 & 0 & 0 \\
2 & & 0 & 1.2915 & 4.6231 & 6.7569 & 4.6310 & 1.2923 & 0 \\
3 & 2.7484 & 4.9056 & 3.9808 & 9.2873 & 3.9841 & 4.8991 & 2.743 \\
4 & 1.6014 & 6.6433 & 9.2900 & 11.5551 & 9.3017 & 6.6538 & 1.601 \\
5 & 2.7469 & 4.9061 & 3.9864 & 9.2902 & 3.9894 & 4.9050 & 2.748 \\
6 & 0 & 1.2931 & 4.6339 & 6.7706 & 4.6391 & 1.2928 & 0 \\
7 & 0 & 0 & 1.1182 & 3.2718 & 1.1188 & 0 & 0 \\
\hline
\end{tabular}

Table 9. Full core radial power (MW) PARCS (normalized).

\begin{tabular}{rrrrrrrr}
\hline $\mathbf{y} \mathbf{x}$ & \multicolumn{1}{c}{$\mathbf{1}$} & \multicolumn{1}{c}{$\mathbf{2}$} & $\mathbf{3}$ & $\mathbf{4}$ & $\mathbf{5}$ & $\mathbf{6}$ & \multicolumn{1}{c}{$\mathbf{7}$} \\
\hline 1 & 0 & 0 & 1.1170 & 3.1178 & 1.1170 & 0 & 0 \\
2 & 0 & 1.3034 & 4.6167 & 6.6716 & 4.6167 & 1.3034 & 0 \\
3 & 2.6357 & 4.8800 & 4.1626 & 9.3808 & 4.1626 & 4.8800 & 2.6357 \\
4 & 1.6195 & 6.6067 & 9.3989 & 11.5485 & 9.3989 & 6.6067 & 1.6195 \\
5 & 2.6357 & 4.8800 & 4.1626 & 9.3808 & 4.1626 & 4.8800 & 2.6357 \\
6 & 0 & 1.30335 & 4.6167 & 6.6716 & 4.6167 & 1.3034 & 0 \\
7 & 0 & 0 & 1.1170 & 3.1178 & 1.1170 & 0 & 0 \\
\hline
\end{tabular}

Table 10. Full core radial power difference (in percent).

\begin{tabular}{crrllllr}
\hline $\mathbf{y} \mid \mathbf{x}$ & \multicolumn{1}{c}{1} & \multicolumn{1}{l}{$\mathbf{2}$} & \multicolumn{1}{l}{$\mathbf{3}$} & \multicolumn{1}{l}{$\mathbf{5}$} & \multicolumn{1}{l}{$\mathbf{5}$} & \multicolumn{1}{c}{$\mathbf{7}$} \\
\hline 1 & 0 & 0 & 0.08 & 4.56 & 0.02 & 0 & 0 \\
2 & 0 & 0.91 & 0.14 & 1.26 & 0.31 & 0.85 & 0 \\
3 & 4.10 & 0.52 & 4.57 & 1.01 & 4.48 & 0.39 & 3.91 \\
4 & 1.13 & 0.55 & 1.17 & 0.06 & 1.05 & 0.71 & 1.15 \\
5 & 4.05 & 0.53 & 4.42 & 0.97 & 4.34 & 0.51 & 4.09 \\
6 & 0 & 0.79 & 0.37 & 1.46 & 0.48 & 0.82 & 0 \\
7 & 0 & 0 & 0.11 & 4.71 & 0.16 & 0 & 0 \\
\hline
\end{tabular}

The maximum value of the difference between Monte Carlo and nodal diffusion approximation in radial full core power distribution is $4.71 \%$. This maximum discrepancy is located in the boundaries of the core.

\section{CONCLUSION}

To some extent, the diffusion approximation still works quite well to analyze the IPWR core model. Even though some discrepancies were observed in the results, the significantly low computational cost is a benefit that cannot be neglected in the diffusion method. Indeed, further improvement in the method used in this work is needed if one wants more accurate results for more reliable analysis.

\section{ACKNOWLEDGMENT}

Both authors would like to acknowledge the Indonesia Endowment Fund of Education (Lembaga Pengelola Dana Pendidikan/LPDP) for funding the authors' education and making this work possible.

\section{AUTHOR CONTRIBUTION}

H. Ardiansyah and M.R. Oktavian equally contributed as the main contributors of this paper. All works related to the research and collecting data were divided equally. Both authors read and approved the final version of the paper.

\section{REFERENCES}

1. Z. Wu, Q. Zhang and H. Abdel-Khalik, Nucl. Technol. 180 (2012) 372.

2. M. R. Oktavian, D. Febrita and M. M. Arief, Cogeneration Power-Desalination in Small Modular Reactors (SMRs) for Load Following in Indonesia, Proceeding of the 2018 4th International Conference on Science and Technology (ICST), Yogyakarta, Indonesia (2018) 1.

3. T. Sainati, G. Locatelli and N. Smith, Energ. Policy 129 (2019) 111.

4. M. A. Elsawi and A. S. B. Hraiz, Nucl. Eng. Des. 293 (2015) 249.

5. S. Choi, K. S. Smith, H. Kim et al., J. Nucl. Sci. Technol. 54 (2017) 705.

6. G. Baiocco, A. Petruzzi, S. Bznuni et al., Ann. Nucl. Energy 107 (2017) 42.

7. F. Fejt and J. Frybort, Ann. Nucl. Energy 117 (2018) 25.

8. E. E. Fridman, J. Leppänen, Revised Methods for Few-Group Cross Section Generation in the Serpent Monte Carlo Code, Proceeding of the PHYSOR 2012, Knoxville, Tennessee (2012).

9. J. Leppänen, M. Pusa, T. Viitanen et al., Ann. Nucl. Energy 82 (2015) 142.

10. J. Leppänen, M. Pusa and E. Fridman, Ann. Nucl. Energy 96 (2016) 126. 
11. A. Wysocki, A. Ward, A. Manera et al., Nucl. Technol. 190 (2015) 323.

12. A. Dambrosio, M. Rušćák, G. Mazzini et al., Ann. Nucl. Energy 117 (2018) 145.

13. A. Sadegh-Noedoost, F. Faghihi, A. Fakhraei et al., Ann. Nucl. Energy 136 (2020) 106995.
14. H. Ardiansyah, A. Agung and A. W. Harto, J. Forum Nukl. 12 (2018) 61.

15. O. Noori-Kalkhoran, A. Minuchehr, R. AkbariJeyhouni et al., Ann. Nucl. Energy 65 (2014) 132.

16. E. Nikitin, E. Fridman and K. Mikityuk, Ann. Nucl. Energy 75 (2015) 492. 